\title{
Size-related assessment on viability and insulin secretion of caprine islets in vitro.
}

\begin{abstract}
The successful isolation, purification, and culture of caprine islets has recently been reported. The present study shows arange of size distribution in caprine islet diameter from 50 to $250 \mu \mathrm{m}$, in which $80 \%$ of the total islet yield was comprised of small islets. Caprine islets were isolated and purified. Islets were handpicked and the diameter of the islets was recorded using light microscopy. Viablility of the islets was analyzed by confocal microscopy. Insulin secretion assay was carried out and analyzed by ELISA. When tested at $48 \mathrm{~h}$ after isolation, these small islets were $29.3 \%$ more viable compared to the large-sized islets. Large islets showed a high ratio (P < $0.01)$ of central core necrosis $(29.5 \% \pm 1.92)$ whilst no significant core death was observed in small islets $(2.33 \% \pm 0.59)$. The annexin assay demonstrated $5.21 \% \pm 0.97$ and $7.34 \% \pm 0.78$ apoptotic death for small and large islets, respectively. During static incubation, small islets released 2.89-fold $(1.39 \pm 0.2 \mathrm{ng} / \mathrm{IE})$ higher insulin level under low glucose induction $(3.3 \mathrm{~mm})$ and simultaneously 2.92-fold $(2.95 \pm 0.33 \mathrm{ng} / \mathrm{IE})$ more insulin under high glucose condition $(16.7 \mathrm{~mm})$ in comparison to large islets at the same islet equivalents $(\mathrm{P}<0.05)$. The present findings evidenced the superior quality of smaller caprine islets compared to larger ones under an optimized basal maintenance condition. As it is equally important to preserve the quality of larger caprine islets, this work warrants further investigation on special culture conditions to support these islets.
\end{abstract}

Keyword: Ccaprine; Functionality; Islets of langerhans; Size viability. 